\title{
Magnetic neutrino scattering by crystals
}

\author{
Jürgen Augustin, Berndt Müller, ${ }^{*}$ and Walter Greiner \\ Institut für Theoretische Physik, Johann-Wolfgang-Goethe-Universität, Postfach 111932, \\ D-6000 Frankfurt am Main 11, West Germany
}

(Received 31 July 1989)

\begin{abstract}
The magnetic dipole scattering of neutrinos by the electrostatic potentials of single atoms as well as crystals is investigated. It is shown that scattering by a rigid cubic lattice can amplify the neutrino-atom cross section by a factor of $N^{1 / 3}, N$ being the number of scatterers. However, comparing the results with typical weak-interaction cross sections, the effect seems to be not observable in experiment.
\end{abstract}

The question whether neutrinos have a magnetic moment $\mu_{v}$ or not is quite topical, especially in the context of the discussion about the solar-neutrino problem. ${ }^{1}$ On the one hand, the available direct measurements give an upper limit of about

$$
\frac{\mu_{v}}{\mu_{0}} \leq 10^{-10}-10^{-9},
$$

where $\mu_{0}$ is the electron Bohr magneton. ${ }^{2}$ On the other hand, an extension of the standard electroweak gauge model to massive neutrinos yields a magnetic moment of

$$
\frac{\mu_{v}}{\mu_{0}}=3.2 \times 10^{-19}\left(\frac{m_{v}}{1 \mathrm{eV}}\right)
$$

due to radiative corrections. ${ }^{2}$ So it would be very useful to find processes which would allow for more accurate measurements of $\mu_{v}$. A possible candidate for such a process is coherent scattering of highly energetic neutrinos on crystals. As it is well known the axial scattering (channeling) of relativistic particles in crystals provides very strong electromagnetic fields in the rest frame of the particle due to the Lorentz contraction of the crystal fields. ${ }^{3}$ Since strongly focused beams are required in such experiments, neutrino channeling is only possible at Superconducting Super Collider energies. The typical transverse energy of about $30 \mathrm{MeV}$ would yield a neutrino beam spread of $6 \mu \mathrm{rad}$ at an energy of $10 \mathrm{TeV}$. Assuming a neutrino mass of the order of $1 \mathrm{eV}$ and neutrino energies in the multi-TeV range, one obtains Lorentz factors of order $\gamma \sim 10^{10}$. Therefore considerable spin-flip amplitudes in neutrino channeling are conceivable despite the small interaction parameter $\mu_{v}$. Given a sufficiently large cross section such a helicity flip could even be observed in elastic collisions, due to the resulting reduction in the number of left-handed neutrinos available for weak interactions downstream. With this idea in mind we investigate the scattering of a neutrino by a cubic array of atoms.

We first evaluate the $T$ matrix for the scattering by one single atom. A massive neutrino moving in an external field is described by the Dirac equation

$$
\left[i \gamma^{\mu} \partial_{\mu}-m_{v}+\frac{\mu_{v}}{2} \sigma^{\mu v} F_{\mu \nu}\right) \psi=0 .
$$

For reasons of simplicity we perform the calculation in the rest frame of the atom, where we do not have to deal with magnetic fields. Separation of the harmonic time dependence $\exp (-i \varepsilon t)$ reduces Eq. (3) to

$$
\left(\boldsymbol{\alpha} \cdot \mathbf{p}+m_{v} \beta-i \mu_{v} \gamma \cdot \mathbf{E}\right) \psi(\mathbf{x})=\varepsilon \psi(\mathbf{x})
$$

For the atomic potentials we use parametrizations of the Thomas-Fermi potential, which were introduced by Lindhard ${ }^{4}$ and Molière ${ }^{5}$ and are often used in channeling calculations. ${ }^{6,7}$ They yield the electric fields, which enter Eq. (4):

$$
\begin{aligned}
& E_{L}(r)=\frac{Z e}{r^{2}}\left[1-\left[1+\frac{3 a^{2}}{r^{2}}\right]^{-3 / 2}\right], \\
& E_{M}(r)=\frac{Z e}{r^{2}} \sum_{i=1}^{3} \alpha_{i} e^{-\beta_{i} r / a}\left[1+\frac{\beta_{i} r}{a}\right) .
\end{aligned}
$$

Here

$$
a=\left(\frac{9 \pi^{2}}{128 Z}\right)^{1 / 3} \frac{\hbar^{2}}{m_{e} e^{2}}
$$

denotes a screening length and

$$
\alpha_{i}=\{0.1,0.55,0.35\}, \quad \beta_{i}=\{6.0,1.2,0.3\}
$$

are fitting parameters. The $T$ matrix is now evaluated in first-order Born approximation, since the interaction Hamiltonian $H_{\text {int }}=-i \mu_{v} \gamma \cdot \mathrm{E}$ is very small. The initial and final states are taken as Dirac plane waves

$$
\varphi(\mathbf{r})=N e^{i \mathbf{k} \cdot \mathbf{r}}\left(\begin{array}{c}
\chi_{1 / 2 m} \\
\frac{\mathbf{p} \cdot \boldsymbol{\sigma}}{\varepsilon+m_{v}} \chi_{1 / 2 m}
\end{array}\right],
$$

which are normalized to delta functions, and eigenfunctions of the helicity operator. In order to consider also inelastic scattering we choose different energy values for the initial and final state: 


$$
\varepsilon_{f}=\varepsilon_{i}+\Delta \varepsilon
$$

The transition matrix element

$$
T_{i f}=\int d^{3} r \varphi_{f}^{\dagger}(\mathbf{r}) H_{\mathrm{int}} \varphi_{i}(\mathbf{r})
$$

$T_{i f}=\left(-i \mu_{v}\right) N_{i} N_{f} \int d^{3} r \chi_{(1 / 2) m_{f}}^{\dagger}\left(\frac{1}{\varepsilon_{i}+m_{v}}\left[\mathbf{E} \cdot \mathbf{p}_{i}+i \boldsymbol{\sigma} \cdot\left(\mathbf{E} \times \mathbf{p}_{i}\right)\right]-\frac{1}{\varepsilon_{f}+m_{v}}\left[\mathbf{E} \cdot \mathbf{p}_{f}+i \boldsymbol{\sigma} \cdot\left(\mathbf{p}_{f} \times \mathbf{E}\right)\right]\right) \chi_{(1 / 2) m_{i}} e^{i\left(\mathbf{k}_{i}-\mathbf{k}_{f}\right) \cdot \mathbf{r}}$

The values $m_{i}$ and $m_{f}$ denote spin projections of the neutrino before and after the collision, respectively. The integrations are conveniently carried out in spherical coordinates, choosing the $z$ axis in the direction of the incident neutrino and the $x-z$ plane as the scattering plane. We abbreviate the radial matrix element by

$$
R(q)=\frac{1}{Z e a} \int_{0}^{\infty} d r r^{2} E(r) j_{1}(q r),
$$

where $q=\left|\mathbf{k}_{i}-\mathbf{k}_{f}\right|$ denotes the momentum transfer. For the Lindhard (5) and the Molière field (6) the integral can be performed analytically:

$$
\begin{aligned}
& R_{L}(q)=\frac{1}{q a}-\sqrt{3} K_{1}(\sqrt{3} q a) \\
& R_{M}(q)=\sum_{i=1}^{3} \alpha_{i} \frac{q a}{\beta_{i}^{2}+(q a)^{2}} .
\end{aligned}
$$

Equations (12) and (13) show that $R(q)$ vanishes for zero momentum transfer, i.e., for forward scattering. $R_{L}(q)$ and $R_{M}(q)$ are plotted in Fig. 1. The results for the spin-flip $(+\rightarrow-)$ and the helicity-conserving $(+\rightarrow+)$ scattering amplitudes are

$$
\begin{aligned}
\left|T_{i f}\right|_{+\rightarrow-}^{2}= & \mu_{v}^{2}\left(N_{i} N_{f}\right)^{2}(4 \pi)^{2} \frac{\left(k_{i}+k_{f}\right)^{2}}{q^{2}} \sin ^{2} \frac{\theta_{f}}{2} \\
& \left.\times \int \frac{p_{i}}{\varepsilon_{i}+m_{v}}+\frac{p_{f}}{\varepsilon_{f}+m_{v}}\right)^{2}(Z e a)^{2} R^{2}, \\
\left|T_{i f}\right|_{+\rightarrow+}^{2}= & \mu_{v}^{2}\left(N_{i} N_{f}\right)^{2}(4 \pi)^{2} \frac{\left(k_{i}-k_{f}\right)^{2}}{q^{2}} \cos ^{2} \frac{\theta_{f}}{2} \\
& \times\left(\frac{p_{i}}{\varepsilon_{i}+m_{v}}-\frac{p_{f}}{\varepsilon_{f}+m_{v}}\right)^{2}(Z e a)^{2} R^{2},
\end{aligned}
$$

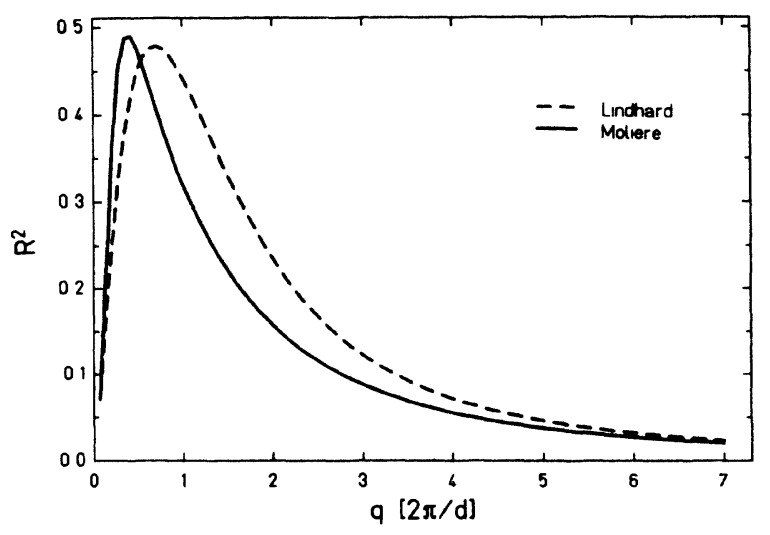

FIG. 1. Radial matrix elements of Eqs. (12) and (13). where $\theta_{f}$ is the scattering angle. For small energy transfers, i.e., $\Delta \varepsilon \ll \varepsilon_{i}$, the helicity-conserving collision is strongly suppressed compared to the spin-flip process and in the elastic channel, $\varepsilon_{i}=\varepsilon_{f}$, the former vanishes completely, i.e., elastic scattering always results in a helicity flip.

It is now a simple matter to construct the differential cross section for the magnetic neutrino-atom scattering from Eqs. (14) and (15). But since we are only interested in an estimate of the order of magnitude for that process, we confine ourselves to the elastic case. When we take into account $q=2 k \sin \left(\theta_{f} / 2\right)$, if $k \equiv k_{i}=k_{f}$, and neglect the indices $i$ and $f$, Eq. (14) reduces to

$$
\left|T_{i f}\right|^{2}=\mu_{v}^{2} N^{4}(Z e a)^{2}(4 \pi)^{2}\left(\frac{2 p}{\varepsilon+m_{v}}\right)^{2} R^{2} .
$$

According to Fermi's "golden rule" the differential cross section that corresponds to the $T$ matrix [(14) and (15)] is given by

$$
d \sigma=2 \pi \frac{d n_{f}}{\phi_{i}} \delta\left(\varepsilon_{f}-\left(\varepsilon_{i}+\Delta \varepsilon\right)\right)\left|T_{i f}\right|^{2} .
$$

The flux $\phi_{i}$ of the incident particle and the momentum density $n_{f} \operatorname{are}^{8}$

$$
\phi_{i}=\frac{2 p_{i} N_{i}^{2}}{\varepsilon_{i}+m_{v}}, \quad d n_{f}=d^{3} k_{f} \frac{\varepsilon_{f}+m_{v}}{2 \varepsilon_{f}(2 \pi)^{3} N_{f}^{2}},
$$

yielding the differential cross section per solid angle,

$$
\frac{d \sigma}{d \Omega_{f}}=\frac{\left(\varepsilon_{i}+m_{v}\right)\left(\varepsilon_{f}+m_{v}\right)}{\left(4 \pi N_{i} N_{f}\right)^{2}} \frac{p_{f}}{p_{i}}\left|T_{i f}\right|^{2},
$$

and the total cross section

$$
\sigma_{\text {tot }}=\mu_{v}^{2}(2 p)^{2} \int d \Omega R^{2} \text {. }
$$

For the Molière matrix element $R_{M}$ the integral can be performed analytically, with the result

$$
\begin{aligned}
& \sigma_{\text {tot }}=2\left(Z e \mu_{v}\right)^{2} \sum_{i, j=1}^{3} \alpha_{i} \alpha_{j} I_{i j}, \\
& I_{i j}=\left\{\begin{array}{l}
\ln \left[1+\left(\frac{2 a k}{\beta_{i}}\right]^{2}\right]-\frac{(2 a k)^{2}}{\beta_{i}^{2}+(2 a k)^{2}} \text { if } i=j, \\
\frac{\beta_{i}^{2}}{\beta_{i}^{2}-\beta_{j}^{2}} \ln \left[1+\left(\frac{2 a k}{\beta_{i}}\right)^{2}\right]+i \leftrightarrow j \text { if } i \neq j .
\end{array}\right.
\end{aligned}
$$

Obviously $\sigma_{\text {tot }}$ exhibits a logarithmic energy dependence. The choice of $Z=32$ (germanium) and $\mu_{v}=10^{-11} \mu_{0}$ yields for a neutrino energy of $10 \mathrm{TeV}$ a cross section of 
the order $10^{-19}$ barns. This number is much below typical neutrino cross sections caused by weak interactions, which grow linearly with the neutrino energy. For example, the total electron-neutrino cross section at $10 \mathrm{TeV}$ due to $W$ - or $Z$-boson exchange has a value of ${ }^{9}$

$$
\sigma_{v_{e^{e^{-}}} \rightarrow v_{e} e^{-}} \approx 10^{-17} \mathrm{~b} \text {. }
$$

Now we shall investigate the question, whether coherent scattering by an oriented crystal leads to a considerable amplification of the total magnetic cross section. We consider only the simplest case, namely, a cubic lattice, assuming that all lattice sites are occupied by identical atoms and neglecting all lattice vibrations. The linear extension of the crystal is $N^{1 / 3} d$, where $d$ is the lattice constant and $N$ the total number of atoms in the array. The positions of the crystal atoms are denoted by vectors $\mathbf{R}_{l}$ :

$$
\begin{aligned}
& \mathbf{R}_{l}=d\left(l_{x} \mathbf{e}_{x}+l_{y} \mathbf{e}_{y}+l_{z} \mathbf{e}_{z}\right), \\
& l_{x}, l_{y}, l_{z}=0,1, \ldots, N^{1 / 3}-1 .
\end{aligned}
$$

The triple of integers $l=\left(l_{x}, l_{y}, l_{z}\right)$ defines a certain atom within the array. The corresponding reciprocal lattice is of the same form, but has the lattice constant $2 \pi / d$. Describing all atoms by the same electric field (5) or (6) the total field of the crystal becomes $\mathbf{E}_{\text {tot }}(\mathbf{r})$ $=\Sigma_{l} \mathbf{E}\left(\mathbf{r}-\mathbf{R}_{l}\right)$, so that Eq. (10) yields

$$
\left|T_{i f}^{\mathrm{tot}}\right|^{2}=\left|T_{i f}\right|^{2}\left|\sum_{l} e^{i \mathbf{q} \cdot \mathbf{R}_{l}}\right|^{2}
$$

Obviously the term $\left|\Sigma_{l} \exp \left(i \mathbf{q} \cdot \mathbf{R}_{l}\right)\right|^{2}$, which contains all effects caused by the lattice, takes the value $N^{2}$, if the momentum transfer $\mathbf{q}$ is equal to any reciprocal lattice vector $\tau_{l}$. For all other values of $q$ it becomes very small, because of destructive interference of the terms in the sum. In fact, this is just the Bragg condition. In order to obtain the total cross section from Eq. (23), we have to integrate over the solid angle:

$$
\sigma_{\mathrm{tot}}=\mu_{v}^{2}(2 p)^{2} \int d \Omega R^{2}\left|\sum_{l} e^{i \mathrm{q} \cdot \mathrm{R}_{l}}\right|^{2} .
$$

To determine, which power of $N$ enters the total cross section, a convenient representation of $\left|\Sigma_{l} \exp \left(i \mathbf{q} \cdot \mathbf{R}_{l}\right)\right|^{2}$ is required. For a cubic crystal the summations are simple geometric progressions and hence we can write ${ }^{10}$

$$
\left|\sum_{l} e^{i \mathbf{q} \cdot \mathbf{R}_{l}}\right|^{2}=\prod_{i=x, y, z} \frac{\sin ^{2}\left(N^{1 / 3} q_{i} \frac{d}{2}\right)}{\sin ^{2}\left(q_{i} \frac{d}{2}\right)} .
$$

This function exhibits the properties mentioned above. It has large peaks of height $N^{2}$, if all components of $\mathrm{q}$ are near an integer multiple of $2 \pi / d$. The width of these peaks is $2 \epsilon$ in each dimension, where $\epsilon=2 \pi / N^{1 / 3} d$ is the distance between a maximum and the following zero of the numerator. To get a form of $(25)$ which can be handled, we approximate the main peaks of the function $\sin ^{2}\left[N^{1 / 3} q_{x}(d / 2)\right] / \sin ^{2}\left[q_{x}(d / 2)\right]$ by triangles of height
$N^{2 / 3}$ and basis width $2 \epsilon$ and neglect the remainder. Averaging this over the peak width then yields, for the three-dimensional case (25),

$$
\left|\sum_{l} e^{i \mathbf{q} \cdot \mathbf{R}_{l}}\right|^{2}=\left\{\begin{array}{l}
\frac{1}{8} N^{2} \text { if } \boldsymbol{\tau}-\boldsymbol{\epsilon}<\mathbf{q}<\boldsymbol{\tau}+\boldsymbol{\epsilon}, \\
0 \text { otherwise } .
\end{array}\right.
$$

Here $\epsilon$ is just the vector $(\epsilon, \epsilon, \epsilon)$. Equation (26) seems to be a crude approximation of the rapidly oscillating function (25), but it still contains its main features. The $q$ integration over a sphere defined by $q_{1}^{2}+\left(q_{z}-k\right)^{2}=k^{2}$, reduces now to a counting problem. Only those reciprocal lattice points contribute to the cross section, that are closer to the sphere than $\epsilon$ (cf. Fig. 2). Since $\epsilon$ is very small compared to the reciprocal lattice constant $2 \pi / d$, the counting cannot be replaced by an integration and becomes quite difficult. But there is a simple way to estimate the cross section. Because of the behavior of the radial matrix element $R$ those reciprocal lattice points contribute most, which correspond to $q$ values of about $2 \pi / d$. There are in the $q_{x}-q_{y}$ plane four of those Bragg points surrounding the $q=0$ point and the minimum radius of the sphere to reach them is

$$
k \geq \frac{\pi}{d} N^{1 / 3} .
$$

Considering the $q^{-2}$ dependence of $R^{2}$ in the asymptotic region the contributions of all other Bragg points are negligible. The cross section obtained in this manner is a lower limit, but it should be close to the exact value.

To give an example we choose the numbers $d=1 \AA$ and $N^{1 / 3}=10^{7}$. This corresponds to a crystal length of 1 $\mathrm{mm}$ and a minimum neutrino energy of $62 \mathrm{GeV}$. $Z$ and $\mu_{v}$ are the same as above. Taking into account a solid angle of

$$
\Delta \Omega=\left(\frac{2 \epsilon}{k}\right)^{2}=\left(\frac{4 \pi}{N^{1 / 3} d}\right)^{2}
$$

for each reciprocal lattice point we obtain from (24) and (26) a total cross section per atom of

$$
\frac{\sigma_{\text {tot }}}{N} \approx N^{1 / 3} \times 4.48 \times 10^{-21} \mathrm{~b}=4.48 \times 10^{-14} \mathrm{~b} .
$$

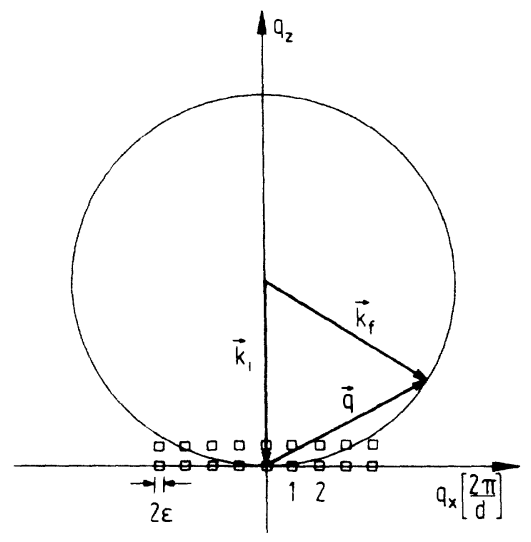

FIG. 2. Solid-angle integration in $q$ space. 
This is only about a factor 50 larger than the electronneutrino cross section due to weak interactions, which is about $10^{-15}$ barn at $60 \mathrm{GeV}$ (Ref. 9). Increasing the energy with $N$ fixed, i.e., taking into account additional Bragg points, does not yield a considerable amplification of the cross section because of the argument given above. At 10 $\mathrm{TeV}, \sigma_{\text {tot }} / N$ is only about 3 or 4 times larger than at 60 $\mathrm{GeV}$. In order to enhance the coherent cross section one has to increase the number of scattering centers $N$ together with the energy.

To summarize, we have calculated the total cross sections for the elastic magnetic neutrino scattering by the electric field of a single atom and a cubic array of atoms. The neutrino-atom scattering turned out to be much smaller than a comparable weak-interaction process, while the neutrino-crystal cross section exhibits an amplification due to coherence. However, we note that this result is strongly based on the assumption of a rigid lattice. If the crystal would pick up a little energy of about $\hbar c \epsilon \approx 10^{-3} \mathrm{eV}$, e.g., by phonon excitation, we would have to integrate over the whole phase space $d^{3} k_{f}$ instead only over $d \Omega_{f}$. $\sigma_{\text {tot }}$ were then proportional to $N$ and the coherence effect would vanish. However, since the elastic cross section exceeds the weak interactions only by one or two orders of magnitude, it is difficult to observe magnetic neutrino scattering in experiment.

We thank Dr. A. Schäfer for helpful discussions.
*Present address: Physics Dept., Duke University, Durham, NC 27706.

${ }^{1}$ M. B. Voloshin, M. I. Vysotskii, and L. B. Okun, Zh. Eksp. Teor. Fiz. 91, 754 (1986) [Sov. Phys. JETP 64, 446 (1986)].

${ }^{2}$ P. Vogel and J. Engel, Phys. Rev. D 39, 3378 (1989).

${ }^{3}$ X. Artru, Phys. Lett. A 128, 302 (1988).

${ }^{4} J$. Lindhard, Mat. Fys. Medd. Dam. Vid. Sesk. 34, 14 (1965).

${ }^{5}$ G. Molière, Z. Naturforsch. A 2, 133 (1947).
${ }^{6}$ D. S. Gemmell, Rev. Mod. Phys. 46, 129 (1974).

${ }^{7}$ J. C. Kimball and N. Cue, Phys. Rep. 125, 69 (1985).

${ }^{8}$ C. J. Joachain, Quantum Collision Theory (North-Holland, Amsterdam, 1983).

${ }^{9}$ P. Becher, M. Böhm, and H. Joos, Eichtheorien (Teubner, Stuttgart, 1981).

${ }^{10} \mathrm{C}$. Kittel, Quantum Theory of Solids (Wiley, New York, 1967). 